\title{
A Newton-Based Extremum Seeking MPPT Method for Photovoltaic Systems with Stochastic Perturbations
}

\author{
Heng Li, ${ }^{1,2}$ Jun Peng, ${ }^{1,2}$ Weirong Liu,, ${ }^{1,2}$ Zhiwu Huang, ${ }^{1,2}$ and Kuo-Chi Lin ${ }^{3}$ \\ ${ }^{1}$ School of Information Science and Engineering, Central South University, Changsha 410075, China \\ ${ }^{2}$ Hunan Engineering Laboratory for Advanced Control and Intelligent Automation, Changsha 410075, China \\ ${ }^{3}$ Department of Mechanical and Aerospace Engineering, University of Central Florida, Orlando, FL 32816, USA
}

Correspondence should be addressed to Jun Peng; pengj@csu.edu.cn

Received 2 May 2014; Revised 6 July 2014; Accepted 6 July 2014; Published 24 July 2014

Academic Editor: Dimitrios Karamanis

Copyright (c) 2014 Heng Li et al. This is an open access article distributed under the Creative Commons Attribution License, which permits unrestricted use, distribution, and reproduction in any medium, provided the original work is properly cited.

\begin{abstract}
Microcontroller based maximum power point tracking (MPPT) has been the most popular MPPT approach in photovoltaic systems due to its high flexibility and efficiency in different photovoltaic systems. It is well known that PV systems typically operate under a range of uncertain environmental parameters and disturbances, which implies that MPPT controllers generally suffer from some unknown stochastic perturbations. To address this issue, a novel Newton-based stochastic extremum seeking MPPT method is proposed. Treating stochastic perturbations as excitation signals, the proposed MPPT controller has a good tolerance of stochastic perturbations in nature. Different from conventional gradient-based extremum seeking MPPT algorithm, the convergence rate of the proposed controller can be totally user-assignable rather than determined by unknown power map. The stability and convergence of the proposed controller are rigorously proved. We further discuss the effects of partial shading and PV module ageing on the proposed controller. Numerical simulations and experiments are conducted to show the effectiveness of the proposed MPPT algorithm.
\end{abstract}

\section{Introduction}

Recent years have seen a growing interest in the research of solar energy, which is mainly due to the advantages solar energy has over traditional fossil energies, including safety, sustainable source of energy, less environment pollution, and numerous market potentials. As the main available solar technology, photovoltaic array has been widely used in satellites and spacecrafts, solar vehicles, and domestic power supply; see $[1,2]$ and the references therein.

The process of guiding a photovoltaic array to its maximum power point is called maximum power point tracking. Due to the inherent nature of photovoltaic cells, the powervoltage curves of PV cells depend nonlinearly on temperature and irradiation intensity; see [3-5]. This fact, however, means that the operating current or voltage that maximizes the output power will change with environmental conditions. To maintain the maximum output power regardless of environmental parameters, one common way is to design MPPT control system to regulate operating current or voltage to the maximum output power point.

With the rapid development of embedded technology, microcontroller based MPPT control system has been the dominated approach in current photovoltaic systems [5]. This approach is favoured because researchers and engineers prefer to program elegant and advanced MPPT algorithms rather than design complicated and expensive MPPT control circuits.

There are a number of MPPT control algorithms that have been proposed in the literature, among which the two primary algorithms are incremental conductance (IncCond) algorithm [5] and perturb and observe (PO) algorithm [6]. In PO algorithms, a step perturbation is added into the control signal and used to monitor the direction of changes in power. PO has been a commercial algorithm because of its ease of implementation. The main drawback of $\mathrm{PO}$ algorithm is that it will oscillate at the maximum power point. Also, it has been shown that PO fails to track the rapidly changing 
irradiance [7]. The IncCond algorithm tracks the maximum power point by comparing the instantaneous and incremental conductances of the PV array. Thus it can track the rapidly changing irradiance. However, the harmonic components of array voltage and current need to be measured and used to adjust the reference voltage, which implies that errors at the maximum power point occur due to the low precision sensors used [8].

A promising new MPPT algorithm is the method of extremum seeking (ES) control; see [9-12]. As a model-free, realtime optimization method, ES is well suited for applications with unknown or partly known dynamics and external perturbations, such as photovoltaic systems [11]. ES uses perturbation signals (either from external disturbances or from converter ripples) as probing signals to estimate the gradient of the power map and then update the control signal according to the estimation [12]. It has been shown that ES has the advantages of both rigorously provable convergence and simplicity of hardware implementation [13]. However, existing extremum seeking MPPT controllers still suffer from the following two limitations.

(i) In existing extremum seeking MPPT controllers, perturbation signals are generally assumed to be periodic. On the one hand, the assumption is rather ideal since external disturbances are typically unknown and stochastic; on the other hand, the requirement of orthogonality makes periodic ES difficult to extend to multivariable cases.

(ii) In existing extremum seeking MPPT controllers, the convergence speed is typically defined by the gradient of power map of PV systems, which implies that control systems will be highly influenced by unknown and changing environmental conditions. To construct a practical MPPT control system, the convergence of MPPT controller should be user-assignable rather than being dependent on environmental conditions.

Thus the aim of this paper is to provide an improved extremum seeking MPPT control algorithm to solve these problems. Recent progress in stochastic averaging theory has made it possible to consider more general stochastic perturbations when designing extremum seeking controllers; see $[14,15]$. In [14], Liu and Krstic provide a systematic design of extremum seeking when stochastic perturbations exist and prove the stability of the stochastic extremum seeking via a developed stochastic averaging theory. The theoretical part of this paper is based on the findings of $[14,15]$ and we further propose a Newton-based stochastic extremum seeking MPPT controller considering physical features of photovoltaic systems. We conduct extensive simulations and experiments to show the effectiveness of the proposed control algorithm.

The remainder of the paper is organized as follows. In Section 2, preliminary knowledge about photovoltaic modelling and maximum power point tracking is introduced. In Section 3, a new Newton-based stochastic extremum seeking MPPT controller is designed. The tracking performance of the proposed controller is evaluated in Section 4. We conclude the paper in Section 5.

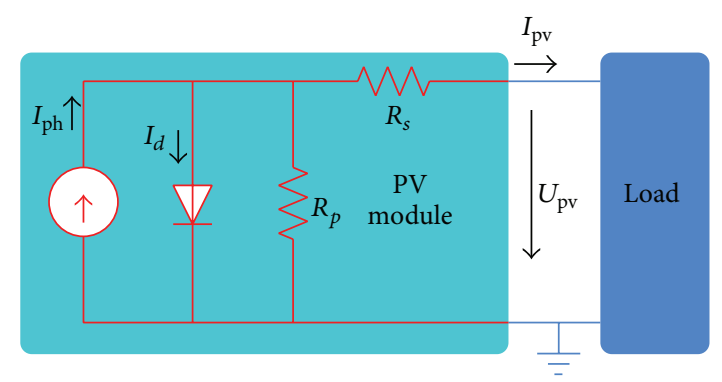

FIGURE 1: The equivalent electrical circuit of a photovoltaic module.

\section{Photovoltaic Modelling and MPPT}

In this section, we will introduce some preliminary knowledge about PV array and MPPT. A photovoltaic array typically consists of several photovoltaic modules connected in series or parallel to obtain the desired output voltage and current. Thus we first introduce the electrical characteristics of PV modules. More detailed background information can be found in [16].

A photovoltaic module can be seen as a black box that produces a current $I_{\mathrm{pv}}$ at a voltage $U_{\mathrm{pv}}$. The inside of that black box can be described by an electric circuit with 4 components, as shown in Figure 1.

(i) Current source: this is the source of the photo current, which can be denoted by

$$
I_{\mathrm{ph}}=S \cdot H \cdot \xi
$$

where $S$ is the module area, $H$ is the intensity of incoming light, and $\xi$ is the response factor.

(ii) Diode: this nonlinear element reflects the dependence on the band gap and losses to recombination. It is characterized by the reverse current $I_{d}$.

(iii) Shunt resistor $R_{p}$ : it represents losses incurred by conductors.

(iv) Serial resistor $R_{s}$ : it also represents losses incurred by nonideal conductors.

The relationship between current $I_{\mathrm{pv}}$ and voltage $U_{\mathrm{pv}}$ of a photovoltaic module is then expressed by

$$
I_{\mathrm{pv}}=I_{\mathrm{ph}}-I_{d}\left[\exp \left(\frac{U_{\mathrm{pv}}+I_{\mathrm{pv}} R_{s}}{U_{T}}\right)-1\right]-\frac{U_{\mathrm{pv}}+I_{\mathrm{pv}} R_{s}}{R_{p}},
$$

where $U_{T}=q k T / e$ with ideality factor $q$, temperature $T$, Boltzmann constant $k=1.38 e^{-23}$, and the elementary charge $e=1.602 e^{-19}$.

Then the output power $P_{\mathrm{pv}}$ of a photovoltaic module is denoted by

$$
P_{\mathrm{pv}}=U_{\mathrm{pv}} I_{\mathrm{pv}}=f\left(U_{\mathrm{pv}}\right),
$$

where function $f(\cdot)$ is determined by manufacturing and environmental parameters, such as module area $S$, irradiance 


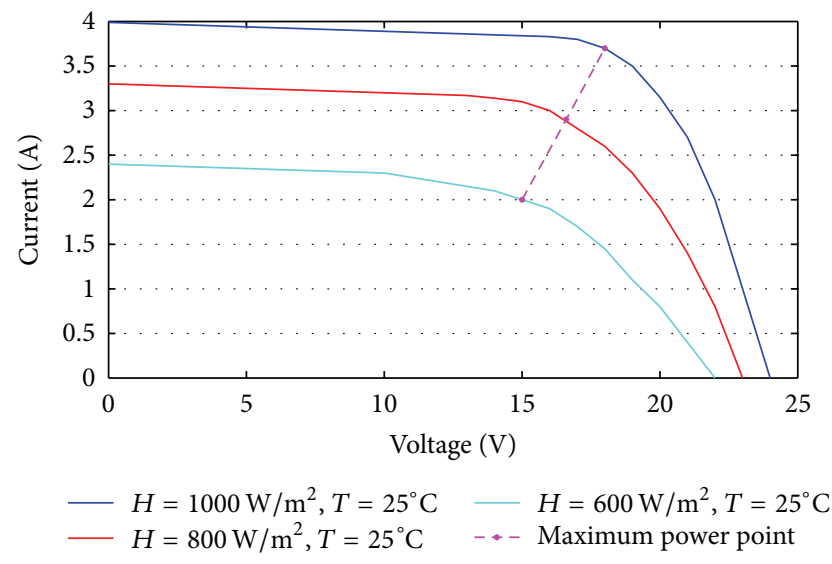

FIGURE 2: $I-V$ characteristics of KC65T PV module at various irradiance levels.

$H$, response factor $\xi$, and temperature $T$, and thus is generally unknown or partially known a priori.

Figure 2 shows the $I-V$ characteristics of the KC65T photovoltaic module at various irradiance levels; see the datasheet of KC65T [18]. With light varying its intensity throughout the day, the maximum power point moves to different voltages and currents. Thus a MPPT control system is typically adopted between the photovoltaic module and the load to adjust the output voltage or current and maintain the maximum power output.

The schematic of a photovoltaic MPPT control system with stochastic perturbations is shown in Figure 3. The photovoltaic panel converts solar energy to electrical energy via the photovoltaic effect. A MPPT control system is designed between the photovoltaic panel and load to optimize the conversion efficiency. The adopted MPPT control system consists of three components: MPPT controller, DC-DC driver, and DC-DC converter. The proposed MPPT system can be either voltage control or current control depending on which one is used as the control variable of the MPPT controller. In this paper, we design the MPPT control system with voltage control, but the proposed method is also suited to current control cases.

As the DC-DC driver and converter are relatively mature in electrical industry, the main work in MPPT control system is the design of MPPT control algorithm. Such a research interest has continued for decades, since even small improvements in performance can lead to great economic benefits. Though considerable progress has been made in this field, the following two problems have not been effectively solved:

(i) how to optimize the output power with unknown power map when stochastic external disturbances exist;

(ii) how to assign the convergence speed by designers rather than unknown power map.

\section{MPPT Controller Design}

In this section, we propose a new Newton-based stochastic extremum seeking MPPT controller to handle the above two difficulties. The proposed controller not only inherits the advantages of classical extremum seeking in model-free optimization but also shows its distinctive features in dealing with stochastic perturbations and assigning convergence rates. In what follows, we will introduce the control structure, implementation, and stability analysis of the proposed controller. Some further discussions will be provided in Section 3.5.

3.1. Controller Structure. In order to illustrate the motivation of designing Newton-based extremum seeking MPPT controller, we first introduce the application of classical gradientbased extremum seeking in MPPT of photovoltaic systems. Figure 4 shows the control structure of a gradient-based extremum seeking MPPT controller.

As shown in Figure 4, $\tau$ is the duty cycle of PWM waves, $\eta$ is the stochastic perturbation, and $\widehat{U}_{\mathrm{pv}}$ is the estimation of desired voltage that optimizes the power map. The model of DC-DC driver and converter can be simplified as follows:

$$
\begin{gathered}
\tau=g\left(U_{\mathrm{pv}}, \widehat{U}_{\mathrm{pv}}\right), \\
U_{\mathrm{pv}}=\ell(\tau) .
\end{gathered}
$$

The maximum power point (MPP) of the photovoltaic system is defined as

$$
P_{\mathrm{pv}}^{*}=f\left(U_{\mathrm{pv}}^{*}\right)=f\left(\ell\left(\tau^{*}\right)\right) \triangleq \max P_{\mathrm{pv}},
$$

where $U_{\mathrm{pv}}^{*}, \tau^{*}$ are the desired voltage and duty cycle at the MPP.

We then denote the voltage estimation error $\widetilde{U}_{\mathrm{pv}}$ by

$$
\widetilde{U}_{\mathrm{pv}}=\widehat{U}_{\mathrm{pv}}-U_{\mathrm{pv}}^{*} .
$$

For the purpose of illustration, assume that the power map $f(\cdot)$ is of the quadratic form; then the averaged system is obtained by

$$
\dot{\tilde{U}}_{\mathrm{pv}}=k \mathrm{H} \widetilde{U}_{\mathrm{pv}},
$$

where $\mathrm{H}$ is the second derivative (Hessian) of the power map. Equation (8) shows that the convergence rate is governed by the unknown Hessian $\mathrm{H}$, which is highly influenced by environmental conditions, such as irradiance and temperature.

A practical MPPT control system often requires that the convergence of the controller should be designer-assignable. We next show that this goal can be realized with the Newtonbased extremum seeking.

If the power map is known, the following Newton optimization algorithm can be used to find the maximum power point:

$$
\frac{d P_{\mathrm{pv}}}{d t}=-\left(\frac{d^{2} f\left(U_{\mathrm{pv}}\right)}{d U_{\mathrm{pv}}^{2}}\right)^{-1} \frac{d f\left(U_{\mathrm{pv}}\right)}{d U_{\mathrm{pv}}} .
$$




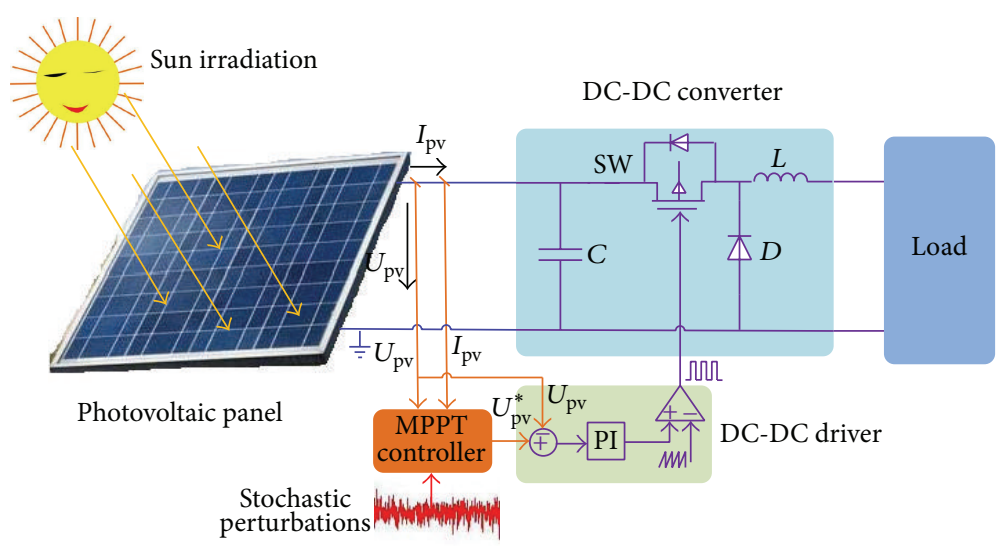

FIGURE 3: The schematic of a photovoltaic MPPT control system with stochastic perturbations.

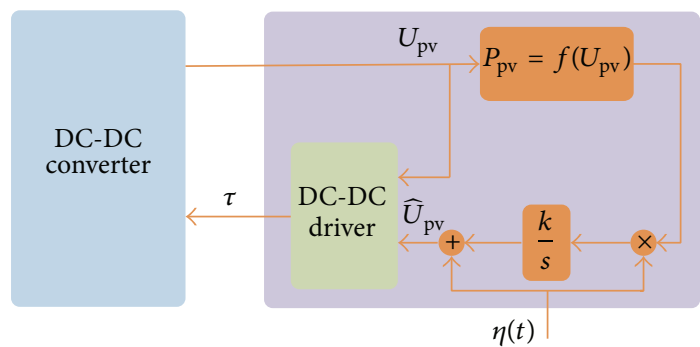

FIGURE 4: The structure of gradient-based extremum seeking MPPT controller.

If $f(\cdot)$ is unknown, then an estimator is needed to approximate $d f(x) / d x$ and $d^{2} f(x) / d x^{2}$. Then the purpose of the controller design is to combine the Newton optimization algorithm (9) with estimators of the first and second derivatives of power map to achieve the maximum power point tracking.

Let $\widehat{G}$ be the estimation of the first-order derivative, $\widehat{H}$ the estimation of the second-order derivative, and $\Gamma$ the estimation of inverse of second derivative. Then we propose a Newton-based stochastic extremum seeking MPPT controller in Figure 5. As shown in Figure 5, the first-order derivative of $f$ is estimated by $\widehat{G}=P_{\mathrm{pv}} M(\eta)$ and the secondorder derivative of $f$ is estimated by $\widehat{\mathrm{H}}=P_{\mathrm{pv}} N(\eta)$. It is typically difficult to derive $\Gamma=1 / \widehat{\mathrm{H}}$ directly when $\widehat{\mathrm{H}}$ is close to zero. Instead, a dynamic estimator $\dot{\Xi}=-h \Xi+h \widehat{\mathrm{H}}$ is employed to approximate $\Gamma$.

We rewrite the proposed control algorithm in state space as

$$
\begin{gathered}
U_{\mathrm{pv}}=g(\tau), \\
\tau=\ell\left(U_{\mathrm{pv}}, \widehat{U}_{\mathrm{pv}}\right), \\
\dot{\widehat{U}}_{\mathrm{pv}}=-k \Gamma M(\eta) P_{\mathrm{pv}}, \\
\dot{\Gamma}=h \Gamma-h \Gamma^{2} N(\eta) P_{\mathrm{pv}},
\end{gathered}
$$

where $\eta$ is a general stochastic signal and $S(\eta)=a \sin (\eta)$, $M(\eta)$, and $N(\eta)$ are the output of signal generator $\Psi$ with the original signal $\eta$.

Remark 1 . The signal generator outputs $M(\cdot), N(\cdot)$ play an important role in the Newton-based stochastic extremum seeking, since they are used to estimate the first-order and second-order derivative of power map $f(\cdot)$, respectively. Theoretically, they can be any bounded and odd continuous functions. However, considering the practical stability of the photovoltaic system, we choose $M(\cdot), N(\cdot)$ by following the design principle in [19] as

$$
\begin{gathered}
M(\eta)=\frac{1}{a W_{0}(q)} \sin (\eta), \\
N(\eta)=\frac{1}{a^{2} W_{0}^{2}(\sqrt{2} q)}\left(\sin ^{2}(\eta)-W_{0}(q)\right),
\end{gathered}
$$

where variables $W_{0}(q)$ and $W_{1}(q)$ are defined as follows:

$$
\begin{gathered}
W_{0}^{2}(\sqrt{2} q)=2\left(W_{1}(q)-W_{0}^{2}(q)\right), \\
W_{0}(q)=\frac{1}{2}\left(1-e^{-q^{2}}\right), \\
W_{1}(q)=\frac{3}{8}-\frac{1}{2} e^{-q^{2}}+\frac{1}{8} e^{-4 q^{2}} .
\end{gathered}
$$

3.2. Controller Implementation. In this subsection, we provide a detailed flow chart of the implementation of the proposed Newton-based extremum seeking MPPT method, as shown in Figure 6. The basic idea of the controller implementation is that the controller measures the output voltage and current and then computes the output power. Then the first derivative and second derivative of the power map are estimated, respectively, based on the product of output power and stochastic signals. If the termination criterion is satisfied, go to the next iteration. If not, regulate the duty cycle and output voltage to update the output power and go to the next iteration. 


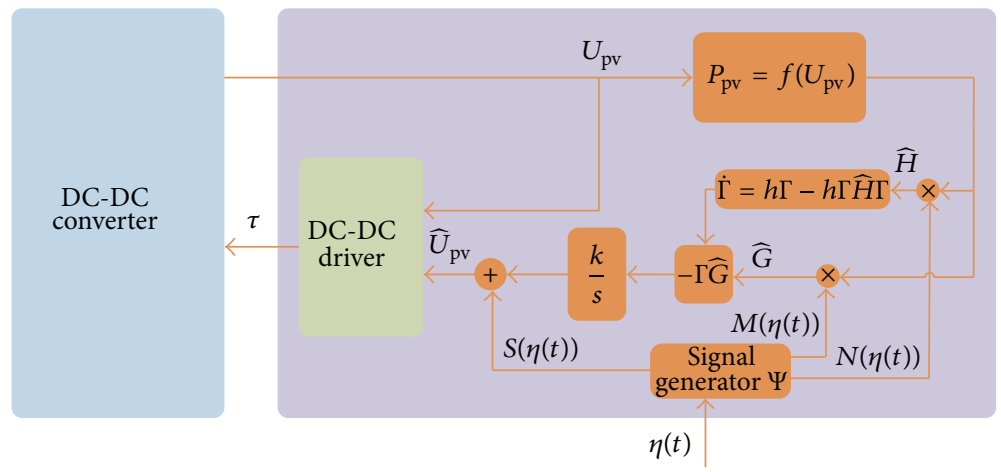

FIGURE 5: The structure of Newton-based extremum seeking MPPT controller.

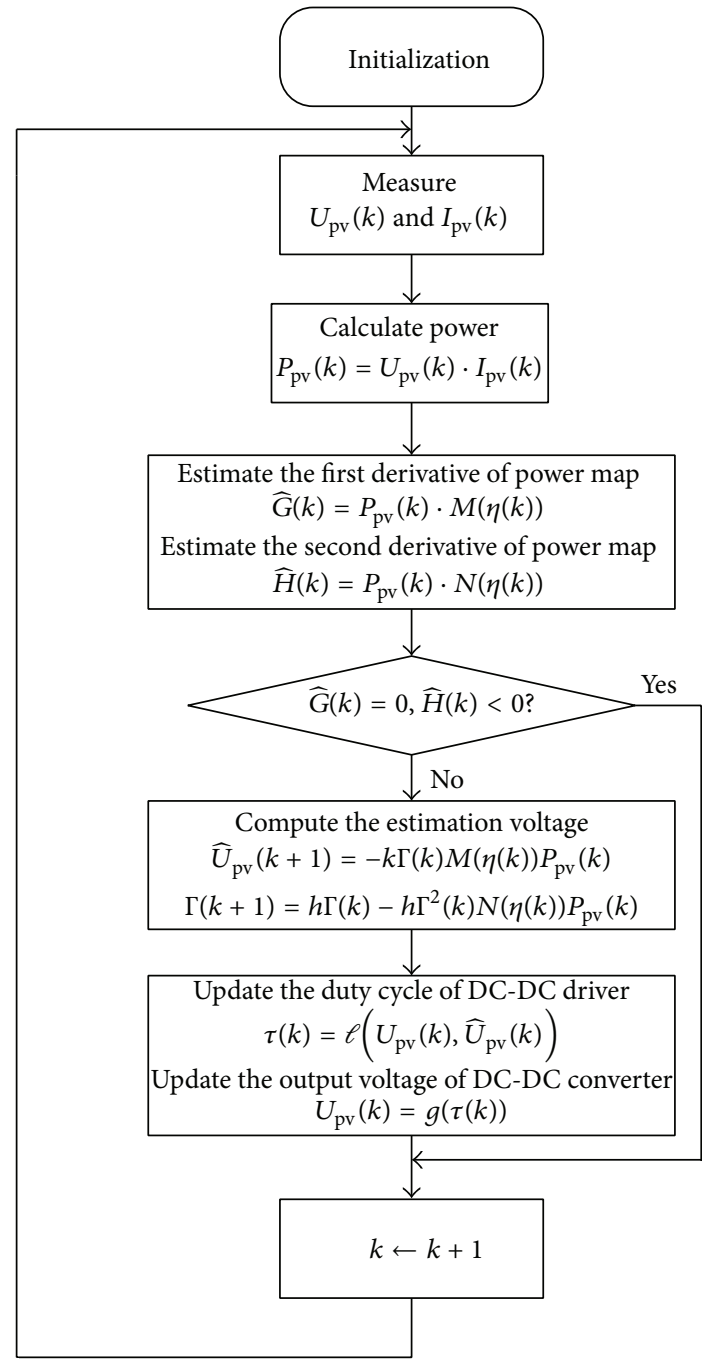

FIGURE 6: Flow chart of the Newton-based extremum seeking MPPT method.

3.3. A Heuristic Analysis. In this subsection, we provide a heuristic analysis of the Newton-based extremum seeking MPPT controller. This illustrative analysis will be helpful

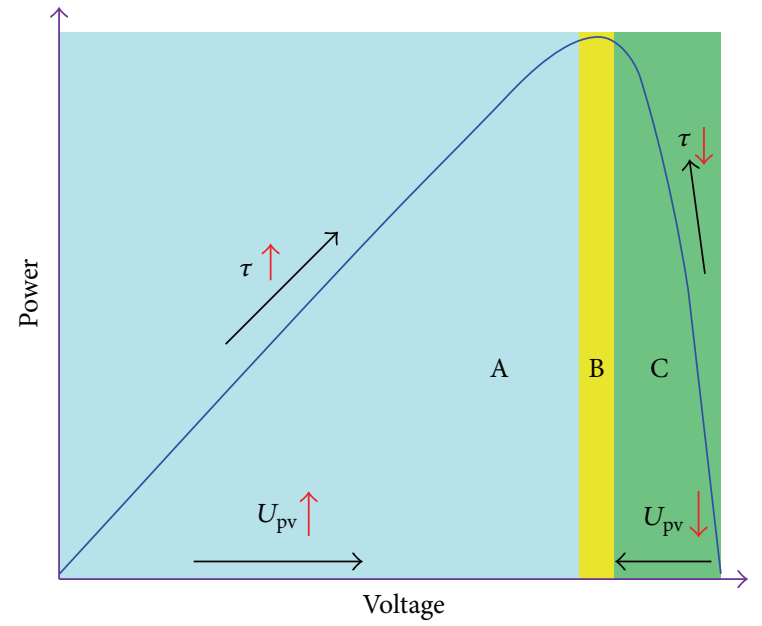

Figure 7: Three different regions of an illustrative $P-V$ curve.

in understanding the proposed MPPT algorithm. A more rigorous stability analysis is provided in Section 3.4.

In the $I-V$ characteristics of a PV module, there are three different regions, namely, current source region, MPP region, and voltage source region [20]. These three regions correspond to the regions $\mathrm{A}, \mathrm{B}$, and $\mathrm{C}$ in the $P-V$ curve, as shown in Figure 7. We will introduce the actions of the MPPT controller in these three regions and explain how the MPPT is achieved.

The update of control signal $\tau$ in (10) can be further simplified as

$$
\tau(k+1)=\tau(k)+\Delta \tau(k),
$$

where $\Delta \tau(k)$ is the update of the control signal $\tau$ in one iteration.

In what follows, we will introduce the update of control input $\tau$ and output voltage $U_{\mathrm{pv}}$ in the following three different regions. 
Region $A$. In this region, the first derivative of the power map $d P_{\mathrm{pv}} / d U_{\mathrm{pv}}>0$ and the second derivative $d^{2} P_{\mathrm{pv}} / d U_{\mathrm{pv}}^{2}<0$. Then the Newton optimization algorithm (9) turns to be

$$
\frac{d P_{\mathrm{pv}}}{d t}=-\left(\frac{d^{2} f\left(U_{\mathrm{pv}}\right)}{d U_{\mathrm{pv}}^{2}}\right)^{-1} \frac{d f\left(U_{\mathrm{pv}}\right)}{d U_{\mathrm{pv}}}>0,
$$

which means that the update of the control signal $\Delta \tau>0$. Then the duty cycle $\tau$ in (10) is increasing. The larger $\tau$ will drive the DC-DC converter to produce larger voltage $U_{\mathrm{pv}}$ and then $U_{\mathrm{pv}}$ will move in the direction of converging to the desired voltage $U_{\mathrm{pv}}^{*}$.

Region $B$. At the MPP, the first derivative $d P_{\mathrm{pv}} / d U_{\mathrm{pv}}=0$ and the second derivative $d^{2} P_{\mathrm{pv}} / d U_{\mathrm{pv}}^{2}<0$. Then the Newton optimization algorithm (9) is

$$
\frac{d P_{\mathrm{pv}}}{d t}=-\left(\frac{d^{2} f\left(U_{\mathrm{pv}}\right)}{d U_{\mathrm{pv}}^{2}}\right)^{-1} \frac{d f\left(U_{\mathrm{pv}}\right)}{d U_{\mathrm{pv}}}=0,
$$

which means that the change of control signal $\Delta \tau=0$. Then the control input $\tau$ and output voltage $U_{\mathrm{pv}}$ keep stable at the desired $\tau^{*}$ and $U_{\mathrm{pv}}^{*}$, which implies that the MPPT is realized.

Region $C$. In this region, the first derivative of the power map $d P_{\mathrm{pv}} / d U_{\mathrm{pv}}<0$ and the second derivative $d^{2} P_{\mathrm{pv}} / d U_{\mathrm{pv}}^{2}<0$. Then the Newton optimization algorithm (9) turns to be

$$
\frac{d P_{\mathrm{pv}}}{d t}=-\left(\frac{d^{2} f\left(U_{\mathrm{pv}}\right)}{d U_{\mathrm{pv}}^{2}}\right)^{-1} \frac{d f\left(U_{\mathrm{pv}}\right)}{d U_{\mathrm{pv}}}<0,
$$

which means that the change of control signal $\Delta \tau<0$. Then the duty cycle $\tau$ in (10) is decreasing. The smaller $\tau$ will drive the DC-DC converter to produce smaller voltage $U_{\mathrm{pv}}$ and then $U_{\mathrm{pv}}$ will move in the direction of converging to the desired voltage $U_{\mathrm{pv}}^{*}$.

3.4. Stability Analysis. Stability is a basic requirement of a practical control system. In this subsection, we analyze the stability of the proposed MPPT algorithm using stochastic averaging theory. Then the main theoretical contribution of this paper is concluded as follows.

Theorem 2. Consider the photovoltaic MPPT control system shown in Figure 3 with DC-DC driver (4), DC-DC converter (5), and unknown power map (3). Assume that the output power $P_{p v}$ reaches its maximum $P_{p v}^{*}$ at the desired control input $\tau^{*}$ and desired voltage $U_{p v}^{*}$. Then under the control law (10) for the update of $\tau$, the stability of the closed-loop system is guaranteed. Moreover, the output power $P_{p v}$ exponentially converges to the maximum power $P_{p v}^{*}$, which implies that the maximum power point tracking is achieved.
Proof. We assume that the stochastic perturbation $\eta(t)$ considered in this paper has invariant distribution $\mu(d x)=$ $(1 / \sqrt{\pi} q) e^{-x^{2} / q^{2}} d x$. Denote the estimation error $\tilde{\tau}=\widehat{\tau}-\tau^{*}$, $\widetilde{\Gamma}=\Gamma-\mathrm{H}^{-1}$. Then the error system can be denoted by

$$
\begin{aligned}
\dot{\tilde{\tau}}= & -k\left(\widetilde{\Gamma}+\mathrm{H}^{-1}\right) M(\eta) f\left(g\left(\tau^{*}+\widetilde{\tau}+a \sin (\eta)\right)\right), \\
\dot{\widetilde{\Gamma}}= & h\left(\widetilde{\Gamma}+\mathrm{H}^{-1}\right) \\
& -h\left(\widetilde{\Gamma}+\mathrm{H}^{-1}\right)^{2} N(\eta) f\left(g\left(\tau^{*}+\widetilde{\tau}+a \sin (\eta)\right)\right) .
\end{aligned}
$$

For simplicity, a quadratic power map is considered:

$$
f(\ell(\tau))=f^{*}+\frac{f^{\prime \prime}\left(\ell\left(\tau^{*}\right)\right)}{2}\left(\tau-\tau^{*}\right)^{2}=f^{*}+\frac{\mathrm{H}}{2}\left(\tau-\tau^{*}\right)^{2} .
$$

Then the error system can be simplified as

$$
\begin{aligned}
\dot{\widetilde{\tau}}= & -k\left(\widetilde{\Gamma}+\mathrm{H}^{-1}\right) M(\eta)\left(f^{*}+\frac{\mathrm{H}}{2}(\widetilde{\tau}+a \sin (\eta))^{2}\right), \\
\dot{\widetilde{\Gamma}}= & h\left(\widetilde{\Gamma}+\mathrm{H}^{-1}\right) \\
& -h\left(\widetilde{\Gamma}+\mathrm{H}^{-1}\right)^{2} N(\eta)\left(f^{*}+\frac{\mathrm{H}}{2}(\widetilde{\tau}+a \sin (\eta))^{2}\right) .
\end{aligned}
$$

To guarantee the stability of closed-loop system, the generated stochastic signals $M(\eta)$ and $N(\eta)$ are chosen as

$$
\begin{gathered}
M(\eta)=\frac{1}{a W_{0}(q)} \sin (\eta), \\
N(\eta)=\frac{1}{a^{2} W_{0}^{2}(\sqrt{2} q)}\left(\sin ^{2}(\eta)-W_{0}(q)\right),
\end{gathered}
$$

where $W_{0}^{2}(\sqrt{2} q)=2\left(W_{1}(q)-W_{0}^{2}(q)\right)$; variables $W_{0}(q)$ and $W_{1}(q)$ are defined as follows:

$$
\begin{gathered}
W_{0}(q)=\frac{1}{2}\left(1-e^{-q^{2}}\right), \\
W_{1}(q)=\frac{3}{8}-\frac{1}{2} e^{-q^{2}}+\frac{1}{8} e^{-4 q^{2}} .
\end{gathered}
$$

Then we obtain the average system

$$
\begin{aligned}
& \dot{\widetilde{\tau}}^{\text {ave }}=-k\left(\widetilde{\tau}^{\text {ave }}-\widetilde{\Gamma}^{\text {ave }} \mathrm{H} \widetilde{\tau}^{\text {ave }}\right), \\
& \dot{\widetilde{\Gamma}}^{\text {ave }}=-h\left(\widetilde{\Gamma}^{\text {ave }}-h\left(\widetilde{\Gamma}^{\text {ave }}\right)^{2} \mathrm{H}\right) .
\end{aligned}
$$

Thus, according to the stochastic averaging theory [14], system (22) has a locally exponentially stable equilibrium at $\left(\widetilde{\tau}^{\text {ave }}, \widetilde{\Gamma}^{\text {ave }}\right)=(0,0)$. When $\widetilde{\tau} \rightarrow 0$, we have $\tau \rightarrow \tau^{*}$ and then $P_{\mathrm{pv}} \rightarrow P_{\mathrm{pv}}^{*}$. Thus the maximum power point tracking is realized. This completes the proof. 
Remark 3. From the equilibrium equation (22), we can find that the convergence of closed-loop system is totally determined by parameters $k$ and $h$, which implies that, in the design of control algorithm (10), the convergence rate can be arbitrarily assigned by the designer with an appropriate choice of $k$ and $h$. Generally speaking, relatively large $k$ and $h$ will lead to a good convergence rate, but too large $k$ and $h$ will also result in oscillations during the convergence. Hence, $k$ and $h$ should be chosen by fully considering both the dynamic and the steady responses.

3.5. Further Discussions. Besides the stability property discussed above, there are some more issues that should be considered in the implementation of MPPT controllers, such as tracking efficiency, effects of partial shading, and PV module ageing effects. In what follows, we will discuss these topics that may be helpful in the application of the proposed MPPT method.

(1) Tracking Efficiency. Tracking efficiency has been the most important consideration of the MPPT method in many commercial applications. The tracking efficiency of a MPPT algorithm can be calculated by the following equation [6]:

$$
\eta_{T}=\frac{\int_{0}^{t} P_{\mathrm{pv}}(t) d t}{\int_{0}^{t} P_{\max }(t) d t},
$$

where $P_{\mathrm{pv}}(t)$ is the measured power produced by the PV array under the control of MPPT algorithm and $P_{\max }(t)$ is the theoretical maximum power that the array can produce.

The discrete definition of the tracking efficiency can be denoted by [21]

$$
\eta_{T}=\frac{1}{n} \sum_{k=0}^{n} \frac{P_{\mathrm{pv}}(k)}{P_{\max }(k)}
$$

where $n$ is the number of samples.

Tracking efficiency evaluates the overall performance of a MPPT algorithm. In the next section, we will verify the tracking efficiency of the proposed MPPT method with numerical results.

(2) Effects of Partial Shading. The partial shading effect has received much attention recently in the implementation of MPPT controller, which is partly due to the rapid development of building integrated photovoltaics (BIPV) [22]. As the BIPV is commonly installed on the rooftop, it typically suffers from partial shading due to the space limitation, clouds, and so forth.

When a PV system is subjected to partial shading, the $P$ $V$ curve often exhibits a global extremum and several local extremums, as shown in Figure 8. Hence, in order to track the global MPP of power map, the adopted MPPT algorithm should have a global convergence. However, from (22) we find that the proposed controller is locally stable at the MPP, which implies that it may get trapped at local extremums and may not be a good choice for PV systems under partial shading. Fortunately, several global or semiglobal extremum

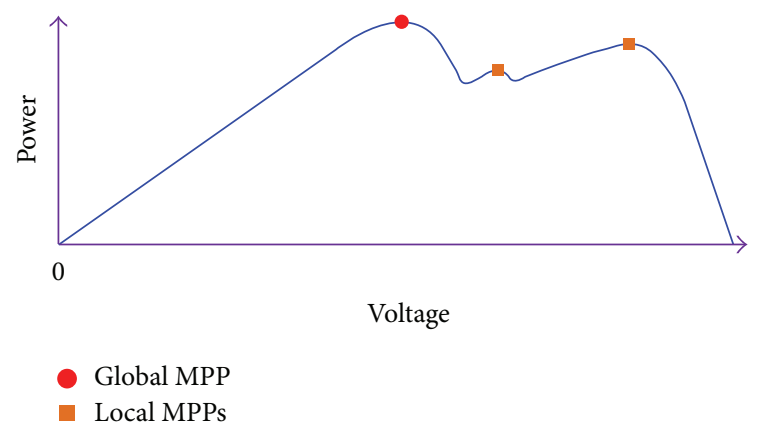

FIgURE 8: An illustrative $P-V$ curve for PV systems under partial shading.

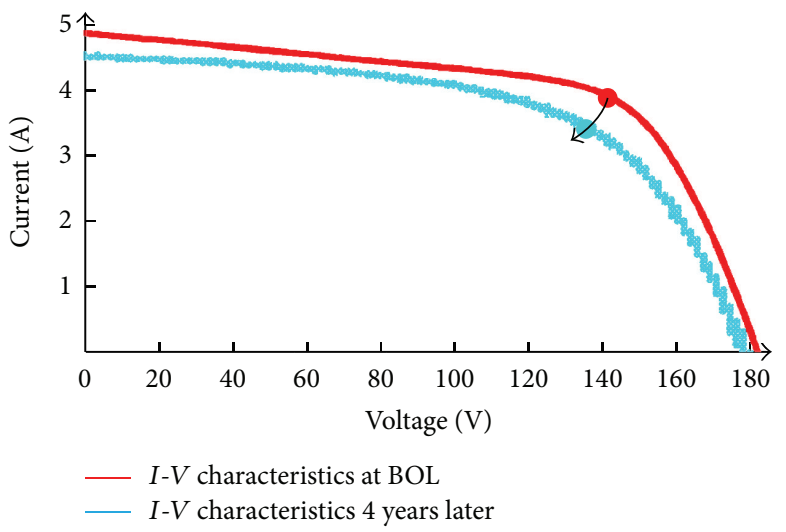

FIGURE 9: Degradation of 20 a-Si modules after 4 years of operation in [17].

seeking control designs have been available; see [23-25] for more information.

(3) Effects of PV Module Ageing. Reliability and lifetime of PV modules are key factors to PV system performance and are mainly dominated by the PV module ageing effect [26]. In order to separate the ageing effect from the irradiance and temperature, it is necessary to evaluate the Beginning of Life (BOL) performance. Then the deviations between the experimental data and BOL performance explain the ageing of the PV system [17].

Figure 9 shows the degradation of 20 a-Si modules after 4 years of operation in the literature [17]. We can find that the MPP declines slowly during the 4-year time. Mathematically, the maximum power point tracking for an ageing PV system is essentially an optimization process for a slowly varying unknown cost function [13]. The proposed Newton-based extremum seeking MPPT controller optimizes the power map with real-time measurements and calls for no knowledge of model information. Thus it can deal with the PV module ageing effect well.

\section{Tracking Performance Evaluation}

In this section, we provide several simulation and experiment results to show the effectiveness of the proposed MPPT 


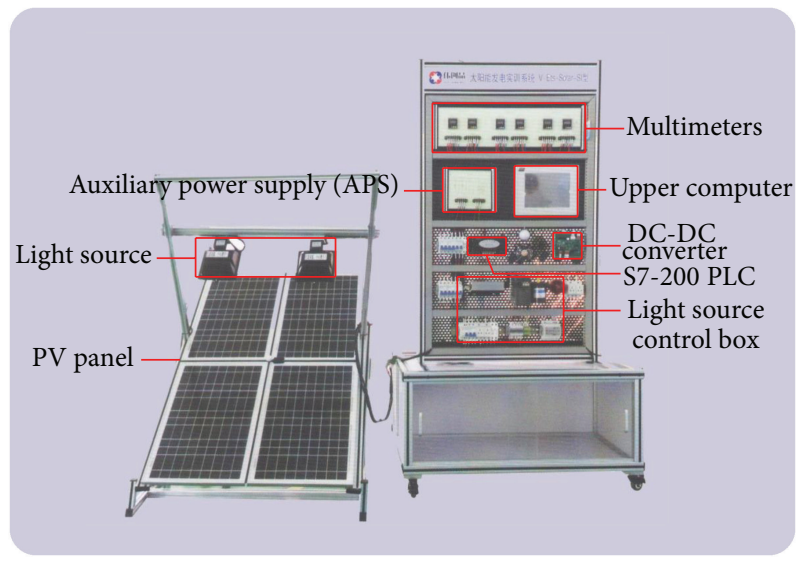

Figure 10: The experiment setup of the light source-driven photovoltaic MPPT system.

algorithm. In order to evaluate the tracking performance of the proposed MPPT algorithm under arbitrary desired irradiance, we adopt the light source-driven photovoltaic MPPT system, as shown in Figure 10.

In Figure 10, there are two incandescents working as the irradiation source. The KC65T-PV panel converts the solar energy to electrical energy via the photovoltaic effect. The Siemens S7-200 PLC works as the MPPT controller, driving the DC-DC converter and regulating the output voltage of the converter. The upper computer is used to collect and store experimental data and multimeters are used to display the measurements. The light source control box is used to regulate the irradiation intensity of light sources.

In what follows, we will provide simulation examples and experiment results to illustrate the tracking performance of the proposed MPPT algorithm under uniform irradiance and nonuniform irradiance cases, respectively. The data sheets of KC65T photovoltaic module under different irradiance conditions are shown in Tables 1 and 2; see [18] for detailed information.

4.1. Tracking under Uniform Irradiance. In this subsection, we consider the tracking performance evaluation of the proposed MPPT algorithm under the uniform irradiance. A simulation and an experiment are provided, respectively, to illustrate the implementation of the algorithm. The simulation is conducted in MATLAB/Simulink and the parameter setting of the proposed control algorithm is shown in Table 3. For simplicity, we adopt only one KC65T PV module in the simulation.

Figure 11 shows the simulation results of the proposed MPPT algorithm under uniform irradiance of $800 \mathrm{~W} / \mathrm{m}^{2}$ at $25^{\circ} \mathrm{C}$. The convergence of the maximum power point is shown in Figure 11(a). The output power oscillates in the direction of MPP which is detected by the product of the power and perturbations. We can see that the output power converges to the MPP within $5 \mathrm{~s}$. The output power in the steady state is about $46.1 \mathrm{~W}$, whereas the maximum power of the PV
TABLE 1: Data sheet of KC65T at $800 \mathrm{~W} / \mathrm{m}^{2}, 25^{\circ} \mathrm{C}$.

\begin{tabular}{ll}
\hline Maximum power $\left(P_{\max }\right)$ & $47.7 \mathrm{~W}$ \\
Maximum power voltage $\left(U_{\text {mpp }}\right)$ & $15.5 \mathrm{~V}$ \\
Maximum power current $\left(I_{\mathrm{mpp}}\right)$ & $3.08 \mathrm{~A}$ \\
\hline
\end{tabular}

TABLE 2: Data sheet of KC65T at $1000 \mathrm{~W} / \mathrm{m}^{2}, 25^{\circ} \mathrm{C}$.

\begin{tabular}{lc}
\hline Maximum power $\left(P_{\max }\right)$ & $65.3 \mathrm{~W}$ \\
Maximum power voltage $\left(U_{\mathrm{mpp}}\right)$ & $17.4 \mathrm{~V}$ \\
Maximum power current $\left(I_{\mathrm{mpp}}\right)$ & $3.75 \mathrm{~A}$ \\
\hline
\end{tabular}

TABLE 3: Parameter setting of the proposed MPPT controller.

\begin{tabular}{lc}
\hline Parameter & Value \\
\hline$a$ & 0.1 \\
$k$ & 1 \\
$h$ & 0.08 \\
$q$ & 40 \\
\hline
\end{tabular}

module is $47.7 \mathrm{~W}$. Then the simulated tracking efficiency of the proposed MPPT algorithm is about $96.6 \%$.

Figure 12 shows the comparison between the proposed Newton-based extremum seeking MPPT method and classical extremum seeking MPPT method. We compare the tracking performance of the two MPPT methods in terms of tracking efficiency and convergence time. The numerical comparison of the two MPPT methods is shown in Table 4.

In addition to the simulations, experiments on the light source-driven photovoltaic MPPT system are conducted for the purpose of verification. The irradiance is set to be $800 \mathrm{~W} / \mathrm{m}^{2}$ and the temperature is about $23 \sim 27^{\circ} \mathrm{C}$. The PV panel comprises four KC65T PV modules, with two modules connected in parallel and two in series.

The experiment results of the proposed MPPT algorithm are shown in Figures 13 and 14. From Figure 13(a), we can see that the output power converges to the MPP within $6 \mathrm{~s}$. The average power at steady state is about $178 \mathrm{~W}$, whereas the maximum power is $190.8 \mathrm{~W}$; then the tracking efficiency is about $93.2 \%$.

4.2. Tracking under Nonuniform Irradiance. In this subsection, we will consider the tracking performance of the proposed MPPT algorithm under nonuniform irradiance with an abrupt change from $800 \mathrm{~W} / \mathrm{m}^{2}$ to $1000 \mathrm{~W} / \mathrm{m}^{2}$ at $10 \mathrm{~s}$. For the purpose of comparison, we also provide the experiment results of the classical gradient-based extremum seeking MPPT method under the same experimental conditions.

Figure 15 shows the experiment results of the proposed Newton-based extremum seeking MPPT method under the nonuniform irradiance. It is shown in Figure 15(a) that the new MPP is located within $2 \mathrm{~s}$. The new steady power is about $243 \mathrm{~W}$, while the maximum power is $261.2 \mathrm{~W}$; then the tracking efficiency is about $93 \%$.

Figure 16 shows the experiment results of the classical gradient-based extremum seeking MPPT method under the 


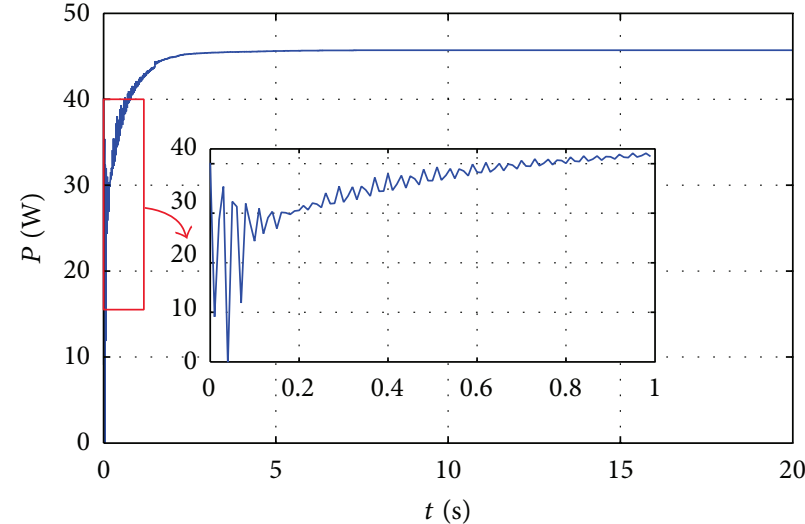

(a) Power

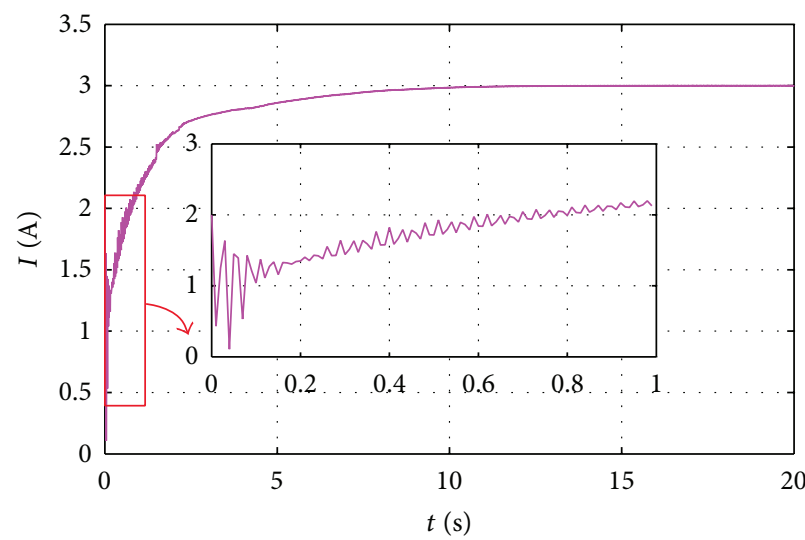

(c) Current

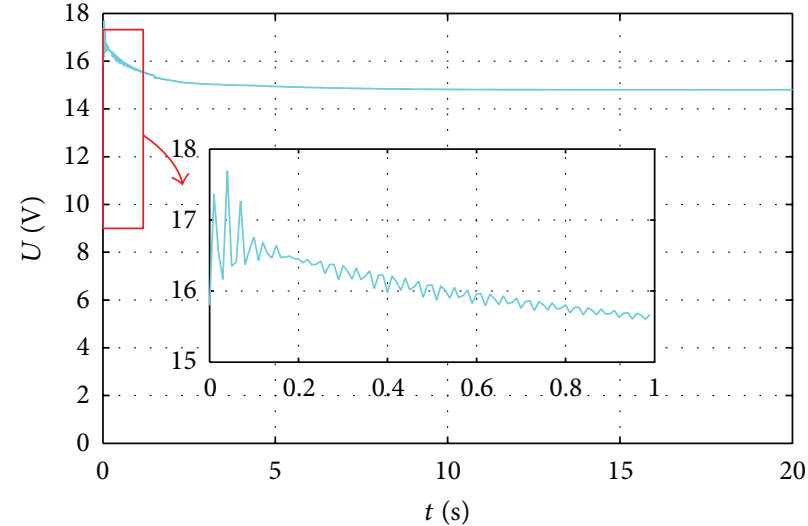

(b) Voltage

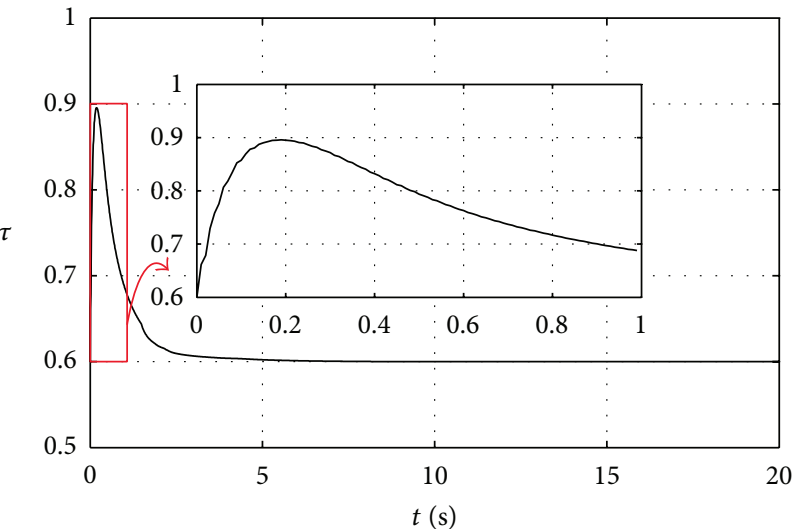

(d) Duty cycle

FIGURE 11: Simulation results of the proposed MPPT method under uniform irradiance of $800 \mathrm{~W} / \mathrm{m}^{2}$ at $25^{\circ} \mathrm{C}$.

TABLE 4: The simulated comparison of the two methods.

\begin{tabular}{lcc}
\hline MPPT method & Efficiency & Convergence \\
\hline Newton-based ES MPPT & $96.6 \%$ & $<4 \mathrm{~s}$ \\
Gradient-based ES MPPT & $92.1 \%$ & $>6 \mathrm{~s}$ \\
\hline
\end{tabular}

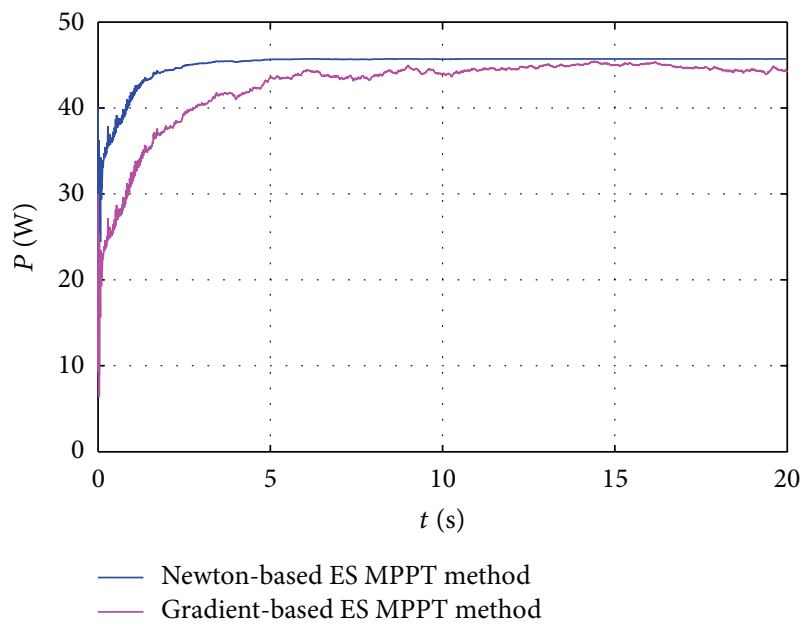

FIGURE 12: The comparison between the proposed MPPT method and classical extremum seeking MPPT method. nonuniform irradiance. It is shown in Figure 16(a) that the new MPP is located within $4 \mathrm{~s}$. The new steady power is about $231 \mathrm{~W}$, while the maximum power is $261.2 \mathrm{~W}$; then the tracking efficiency is about $88.4 \%$.

In order to illustrate the effectiveness of the proposed MPPT method more intuitively, we provide the numerical comparison between the proposed algorithm and classical extremum seeking MPPT algorithm under different irradiance. Figures 17 and 18 show the comparison of the two methods under irradiance $800 \mathrm{~W} / \mathrm{m}^{2}$ and irradiance $1000 \mathrm{~W} / \mathrm{m}^{2}$, respectively. We can see that the proposed method shows its superiority in both tracking efficiency and convergence rate.

\section{Concluding Remark}

In this paper, we propose a new Newton-based extremum seeking MPPT algorithm for photovoltaic systems. The proposed algorithm benefits the following advantages: it calls for no knowledge of the power map; it has a good tolerance of stochastic perturbations and its convergence rate can be totally designer-assignable. The stability and convergence of the proposed MPPT controller are rigorously proved with stochastic averaging theory. Some topics related to applications are discussed, such as partial shading effects and PV 


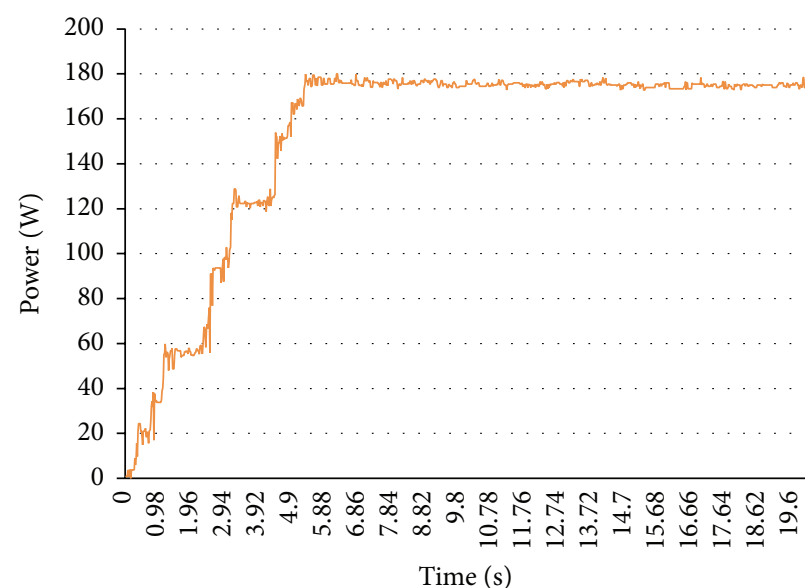

(a) Power

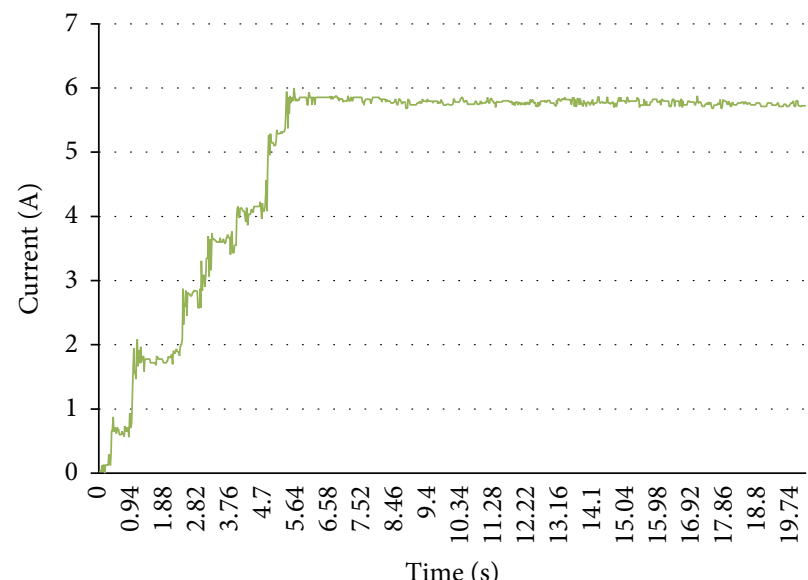

(b) Current

FiguRE 13: Experiment results of output power and current collected in the upper computer.

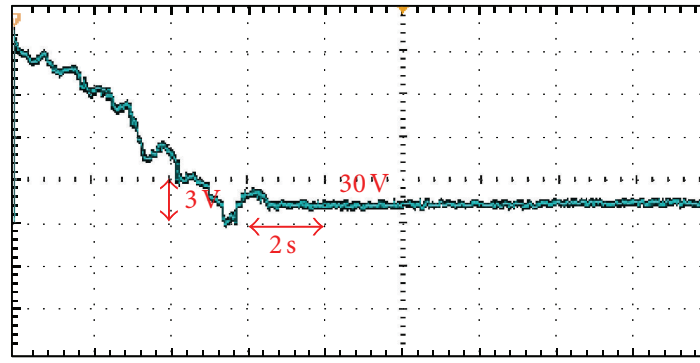

(a) Voltage

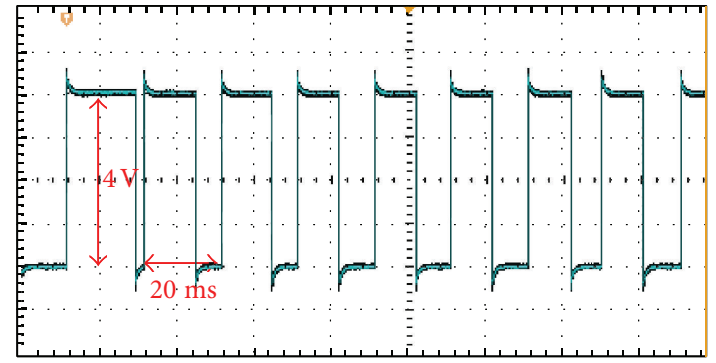

(b) PWM signal

FIGURE 14: Experiment results of output voltage and PWM signal measured in the oscilloscope.

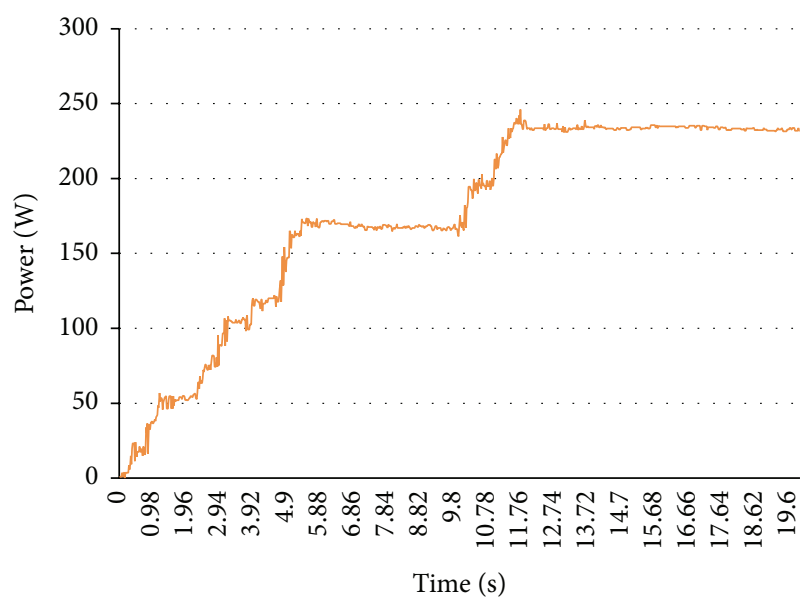

(a) Power

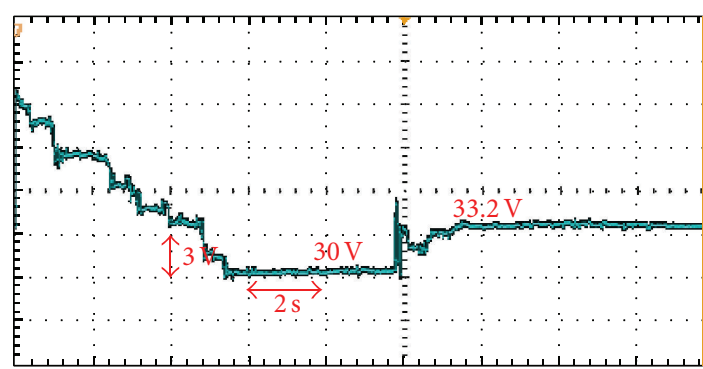

(c) Voltage

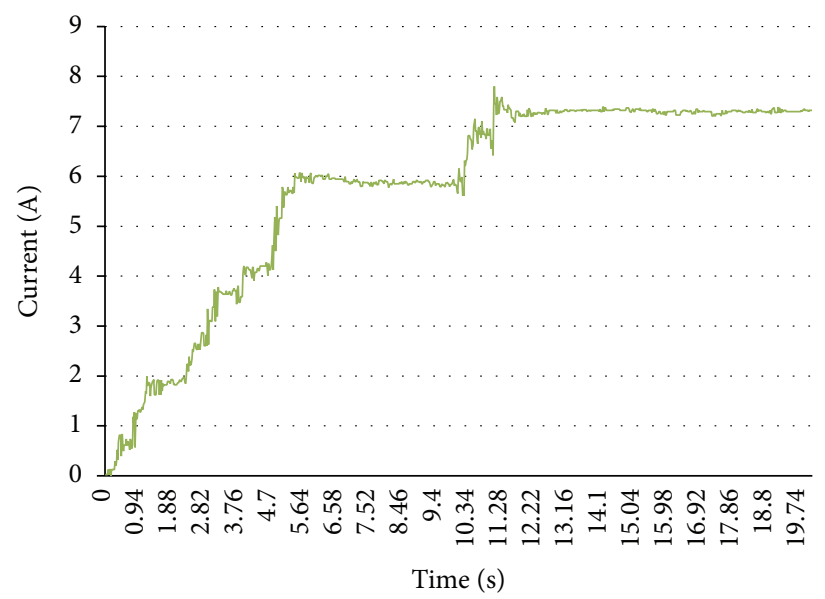

(b) Current

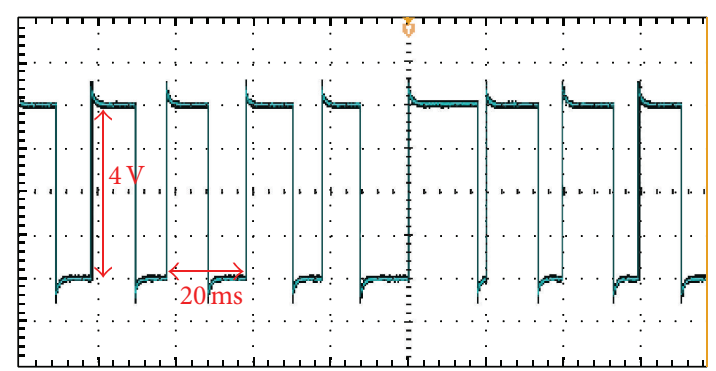

(d) PWM signal

FIGURE 15: Tracking performance of the Newton-based extremum seeking MPPT algorithm under nonuniform irradiance. 


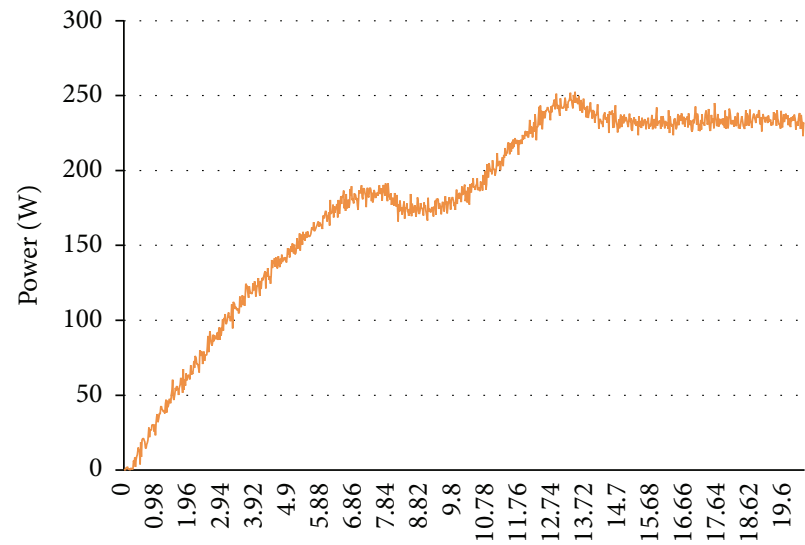

Time (s)

(a) Power

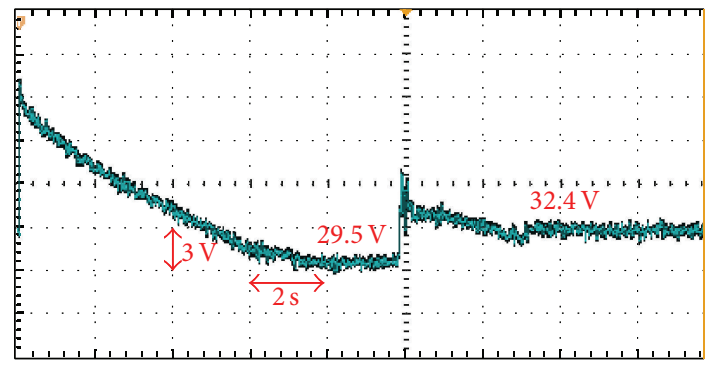

(c) Voltage

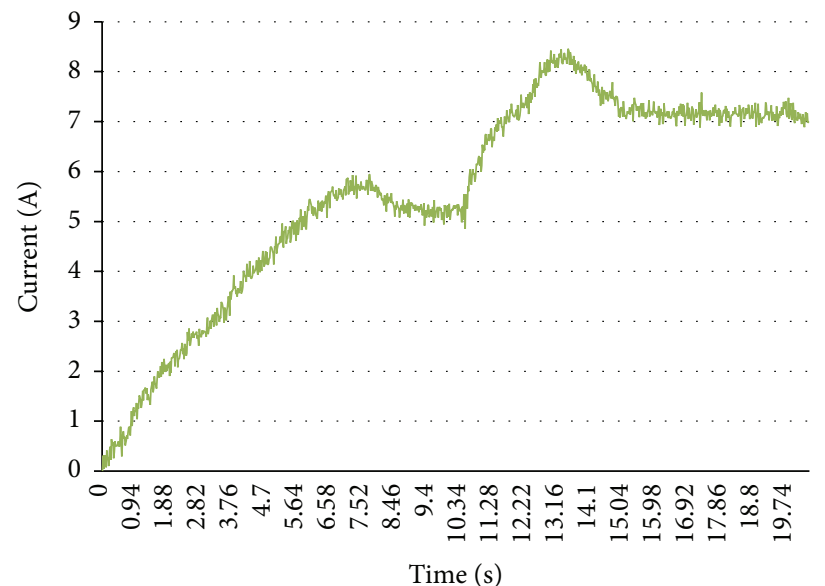

(b) Current

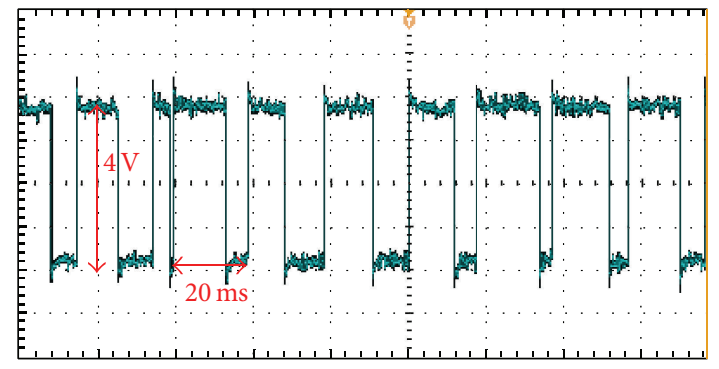

(d) PWM signal

FIGURE 16: Tracking performance of the gradient-based extremum seeking MPPT algorithm under nonuniform irradiance.

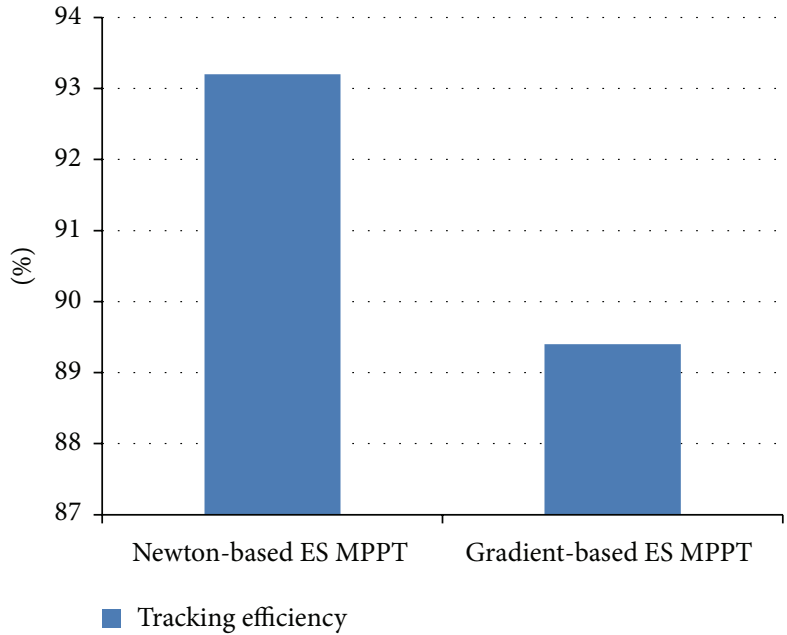

(a) Tracking efficiency

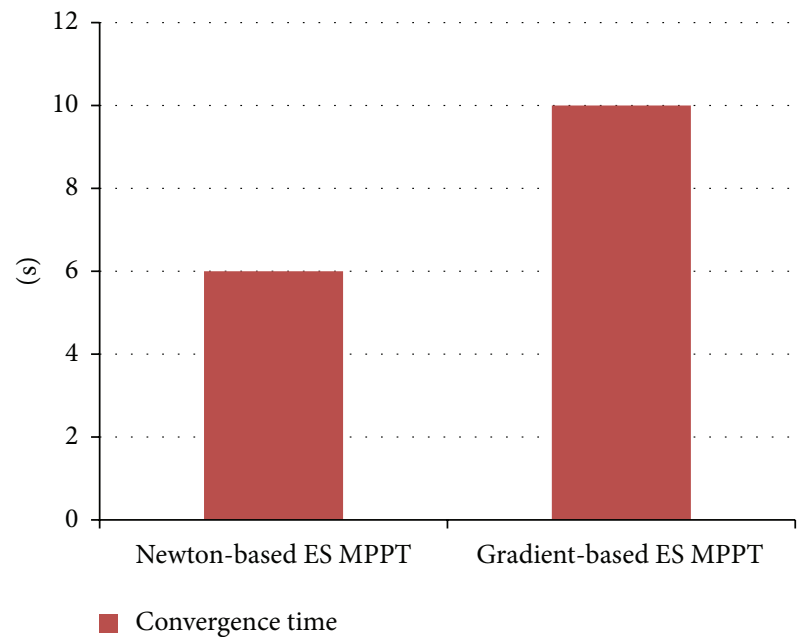

(b) Convergence time

FIGURE 17: The numerical comparison between the proposed MPPT algorithm and classical extremum seeking MPPT algorithm under irradiance $800 \mathrm{~W} / \mathrm{m}^{2}$.

module ageing effects. Simulation and experiment results are provided to show the effectiveness of the proposed method.

It is also worth mentioning that both the Newton-based ES and gradient-based ES belong to the so-called static extremum seeking, which requires that the power map of photovoltaic systems is rather slower than extremum seeking. When irradiance changes quickly, the tracking performance of ES MPPT algorithms will be worse. In order to propose 


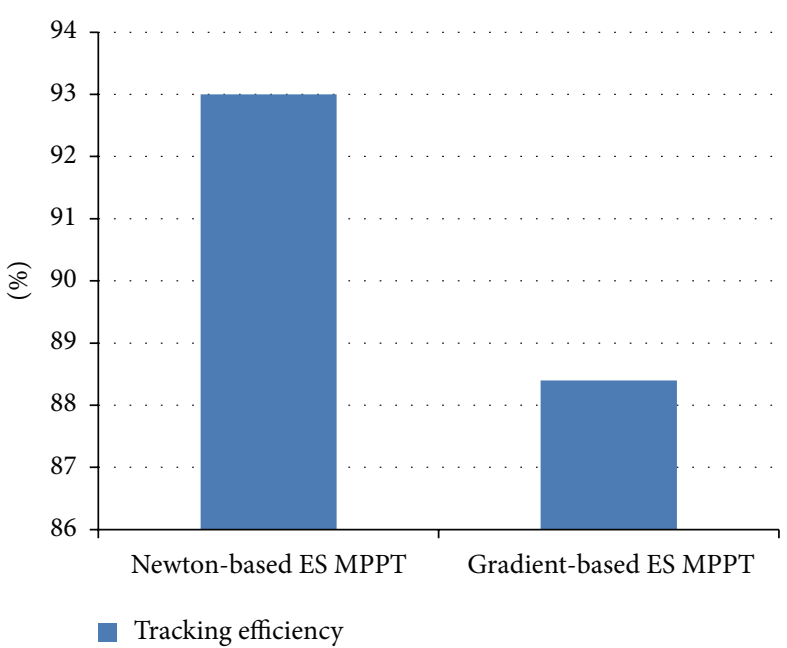

(a) Tracking efficiency

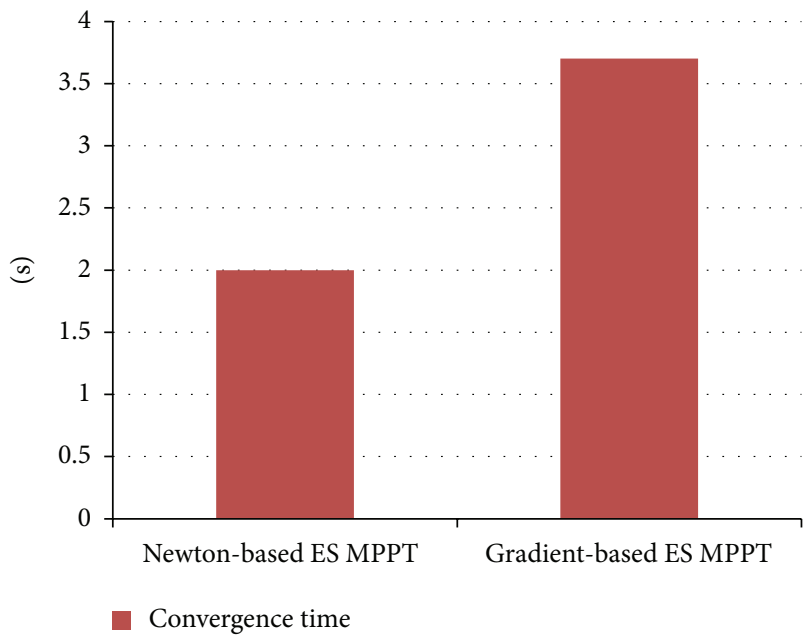

(b) Convergence time

FIGURE 18: The numerical comparison between the proposed MPPT algorithm and classical extremum seeking MPPT algorithm under irradiance $1000 \mathrm{~W} / \mathrm{m}^{2}$.

a commercial ES MPPT algorithm, we will further investigate the dynamic extremum seeking and its applications in maximum power point tracking in future work.

\section{Conflict of Interests}

The authors declare that there is no conflict of interests regarding the publication of this paper.

\section{Acknowledgments}

The authors would like to thank the anonymous reviewers for their valuable comments. This work is supported by the National Natural Science Foundation of China (Grant nos. 61071096 and 61379111), the Fundamental Research Funds for the Central Universities of Central South University, and Hunan Provincial Innovation Foundation for Postgraduate.

\section{References}

[1] E. F. Camacho, M. Berenguel, F. R. Rubio, and D. Martinez, Control of Solar Energy Systems, Springer, 2012.

[2] E. F. Camacho, M. Berenguel, and F. R. Rubio, Advanced Control of Solar Plants, Springer, 1997.

[3] T. Esram and P. L. Chapman, "Comparison of photovoltaic array maximum power point tracking techniques," IEEE Transactions on Energy Conversion, vol. 22, no. 2, pp. 439-449, 2007.

[4] V. Salas, E. Olías, A. Barrado, and A. Lázaro, "Review of the maximum power point tracking algorithms for stand-alone photovoltaic systems," Solar Energy Materials and Solar Cells, vol. 90, no. 11, pp. 1555-1578, 2006.

[5] K. H. Hussein, I. Muta, T. Hoshino, and M. Osakada, "Maximum photovoltaic power tracking: an algorithm for rapidly changing atmospheric conditions," IEE Proceedings: Generation, Transmission and Distribution, vol. 142, no. 1, pp. 59-64, 1995.
[6] D. P. Hohm and M. E. Ropp, "Comparative study of maximum power point tracking algorithms," Progress in Photovoltaics: Research and Applications, vol. 11, no. 1, pp. 47-62, 2003.

[7] T. Kawamura, K. Harada, Y. Ishihara et al., "Analysis of MPPT characteristics in photovoltaic power system," Solar Energy Materials and Solar Cells, vol. 47, no. 1-4, pp. 155-165, 1997.

[8] C. Hua, J. Lin, and C. Shen, "Implementation of a DSPcontrolled photovoltaic system with peak power tracking," IEEE Transactions on Industrial Electronics, vol. 45, no. 1, pp. 99-107, 1998.

[9] R. Leyva, C. Alonso, I. Queinnec, A. Cid-Pastor, D. Lagrange, and L. Martínez-Salamero, "MPPT of photovoltaic systems using extremum-seeking control," IEEE Transactions on Aerospace and Electronic Systems, vol. 42, no. 1, pp. 249-258, 2006.

[10] S. L. Brunton, C. W. Rowley, S. R. Kulkarni, and C. Clarkson, "Maximum power point tracking for photovoltaic optimization using ripple-based extremum seeking control," IEEE Transactions on Power Electronics, vol. 25, no. 10, pp. 2531-2540, 2010.

[11] H. Yau and C. Wu, "Comparison of extremum-seeking control techniques for maximum power point tracking in photovoltaic systems," Energies, vol. 4, no. 12, pp. 2180-2195, 2011.

[12] A. Ghaffari, M. Krstić, and S. Seshagiri, "Extremum seeking for wind and solar energy applications," ASME Dynamic Systems \& Control Magazine, pp. 13-21, 2014.

[13] D. Dochain, M. Perrier, and M. Guay, "Extremum seeking control and its application to process and reaction systems: a survey," Mathematics and Computers in Simulation, vol. 82, no. 3, pp. 369-380, 2011.

[14] S. Liu and M. Krstic, "Stochastic averaging in continuous time and its applications to extremum seeking," IEEE Transactions on Automatic Control, vol. 55, no. 10, pp. 2235-2250, 2010.

[15] S. Liu and M. Krstic, "Newton-based stochastic extremum seeking," Automatica, vol. 50, no. 3, pp. 952-961, 2014.

[16] http://www.greenrhinoenergy.com/solar/.

[17] A. Abete, F. Scapino, F. Spertino, and R. Tommasini, "Ageing effect on the performance of a-Si photovoltaic modules in a grid connected system: experimental data and simulation 
results," in Proceedings of the Conference Record of the 28th IEEE Photovoltaic Specialists Conference, pp. 1587-1590, Anchorage, Alaska, USA, 2000.

[18] http://www.kyocerasolar.com/assets/001/5170.pdf.

[19] S. Liu and M. Krstic, Stochastic Averaging and Stochastic Extremum Seeking, Springer, London, UK, 2012.

[20] P. Bhatnagar and R. K. Nema, "Maximum power point tracking control techniques: state-of-the-art in photovoltaic applications," Renewable and Sustainable Energy Reviews, vol. 23, pp. 224-241, 2013.

[21] A. Reza Reisi, M. Hassan Moradi, and S. Jamasb, "Classification and comparison of maximum power point tracking techniques for photovoltaic system: a review," Renewable and Sustainable Energy Reviews, vol. 19, pp. 433-443, 2013.

[22] K. Ishaque and Z. Salam, "A review of maximum power point tracking techniques of PV system for uniform insolation and partial shading condition," Renewable \& Sustainable Energy Reviews, vol. 19, pp. 475-488, 2013.

[23] Y. Tan, D. Nešić, I. M. Y. Mareels, and A. Astolfi, "On global extremum seeking in the presence of local extrema," Automatica, vol. 45, no. 1, pp. 245-251, 2009.

[24] F. Esmaeilzadeh Azar, M. Perrier, and B. Srinivasan, "A global optimization method based on multi-unit extremum-seeking for scalar nonlinear systems," Computers and Chemical Engineering, vol. 35, no. 3, pp. 456-463, 2011.

[25] W. H. Moase and C. Manzie, "Semi-global stability analysis of observer-based extremum-seeking for Hammerstein plants," IEEE Transactions on Automatic Control, vol. 57, no. 7, pp. 16851695, 2012.

[26] R. Khatri, S. Agarwal, I. Saha, S. K. Singh, and B. Kumar, "Study on long term reliability of photo-voltaic modules and analysis of power degradation using accelerated aging tests and electroluminescence technique," Energy Procedia, vol. 8, pp. 396-401, 2011. 

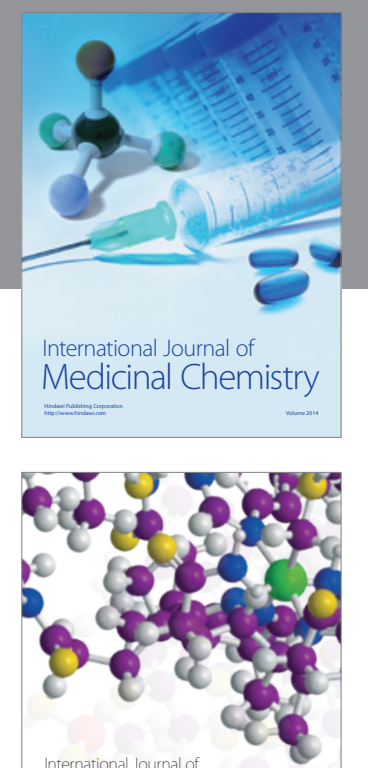

\section{Carbohydrate} Chemistry

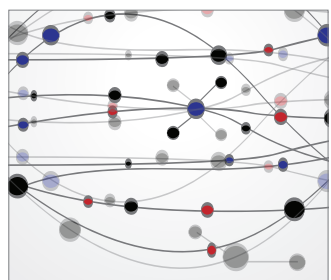

The Scientific World Journal
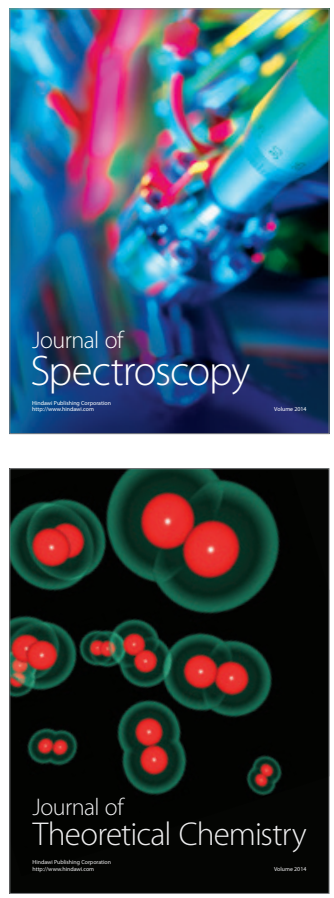
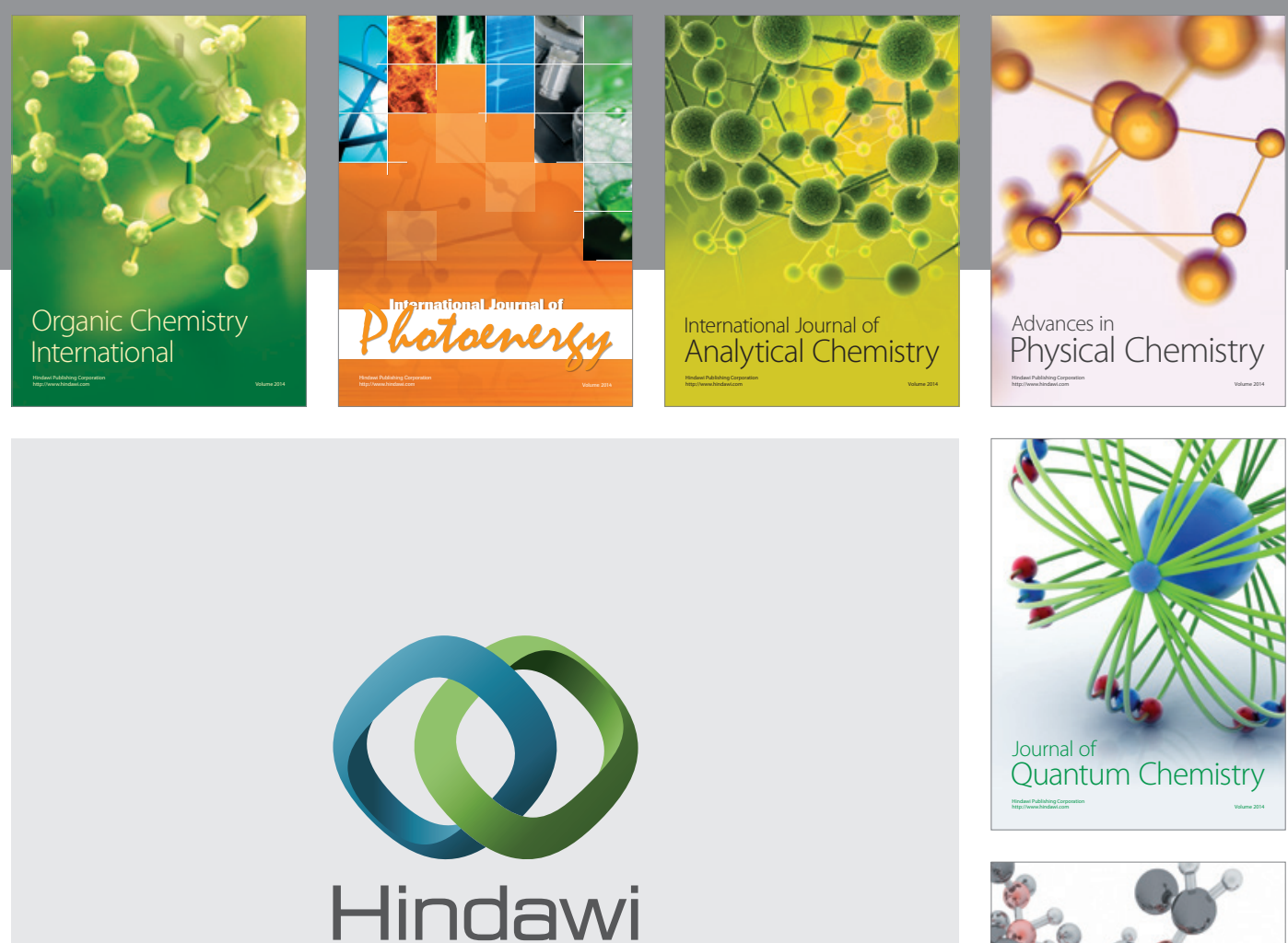

Submit your manuscripts at

http://www.hindawi.com

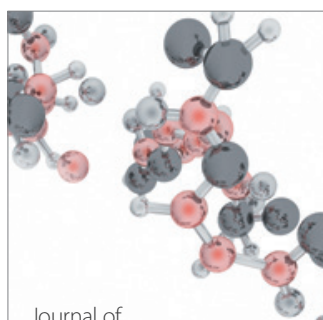

Analytical Methods

in Chemistry

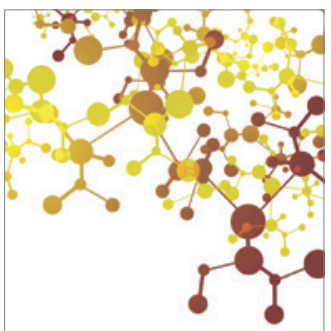

Journal of

Applied Chemistry

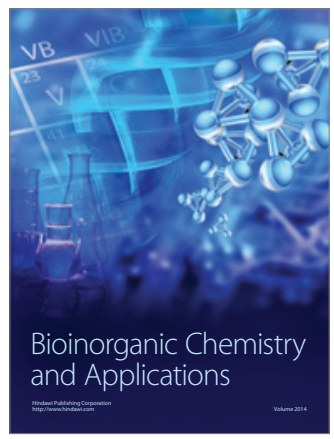

Inorganic Chemistry
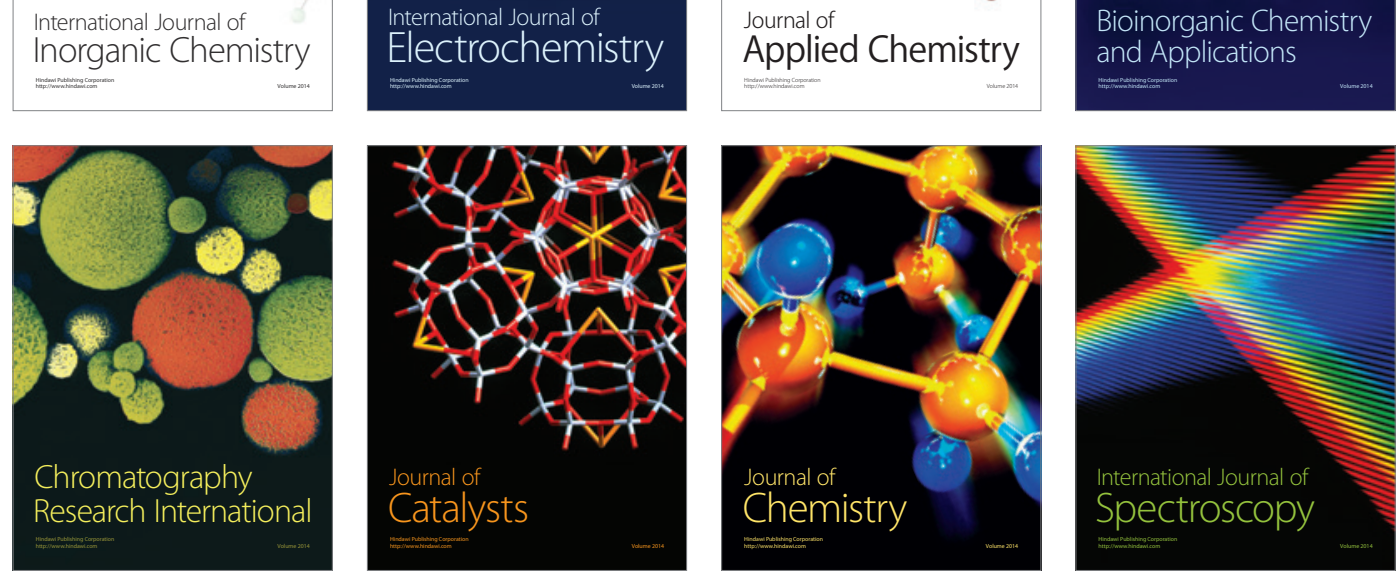